\title{
Comparison of Two Techniques of Reaming for Delayed-Union Long Bone Fractures of Lower Limb - A New Innovative Technique
}

\author{
Dr. Praveen Kumar Pandey ${ }^{1}$, Dr. Jyoti Gupta ${ }^{2}$
}

MS Ortho.

MD Anaesthesia

\begin{abstract}
Background: Delayed union and non-union are complications of fracture healing and remain problematic to treat. Approximately 5\% of all long bone fractures result in delayed union which most often progresses to non-union if not intervened. Delayed union is a potentially chronic condition associated with pain and functional and psychosocial disability. Delayed union is at substantial risk for becoming a non union without further intervention. There is no study available on treatment methods for delayed union of long bone fracture of lower limb including reaming in staged manner. Materials and Methods: A randomized controlled study was conducted in the Department of Orthopaedics, ESI-PGIMSR model hospital, Basaidarapur New delhi. Total of 50 patients diagnosed to have delayed union of factures of long bones such as femur, tibia and fibula were included as cases and divided into two groups on the basis of randomization protocol decided prior to study. In group 1, technique 1 of reaming utilized which includes reaming of proximal fragment first with increasing size of reamer then reaming of distal fragment with increasing size of reamer. In group 2, technique 2 of reaming utilized which is classical technique of reaming both the fragments in sequential manner. Both the groups followed by nailing of adequate sizes and predetermined post-operative protocol. Statistical analysis was done using chi-square test and odds ratio. Results: Both groups were compared for (1) duration from procedure and initiation of bridging callus, and (2) duration from procedure and complete union. Both groups were compared for the (3) number of patients required re-surgery for bone grafting in case of delayed union or non-union after procedure. Group 1 showed mean duration of 2 months from procedure and initiation of bridging callus and mean duration of 10 months from procedure and complete union. Only 5 patients in group 1 required re-surgery for bone grafting. While in group 2, mean duration from procedure and initiation of bridging callus found to be 3 months and mean duration from procedure and complete union found to be 12 months. 13 patients in group 2 required re-surgery for bone grafting. There is clinically significant difference found between two groups in terms of re-surgery for bone grafting. Discussion: Inadequate treatment of a fracture can result in delayed union. The ultimate goal of treating surgeon is to promote fracture healing and prevent complications like delayed union and non union. In our study we found better results with technique one of doing the reaming of proximal fragment first with increasing size of reamer then reaming of distal fragment with increasing size of reamer than technique two of reaming both the fragments in sequential manner in terms of speed of bone healing and recovery.
\end{abstract}

Keywords: delayed union; reaming; sequential; non-union; re-surgery

\section{Introduction}

Fracture healing becomes problematic for treating orthopaedists when this is complicated by delayed union/ non-union. This complication is seen by orthopaedists very commonly now-a-days in day to day OPD. Delayed union of a long bone fracture is defined as a fracture not healed over a period of time that would be adequate for healing of the fracture in that region and is at risk to go into non-union if not intervened. Delayed union and non-union of a long bone should be considered as a continuum when the patient is not intervened by treating orthopaedists at adequate time. 90- 95 $\%$ of all fractures of long bones usually heal with-/ without posing any problem to the treating orthopaedist.[1,2] While considering delayed union, this comprises of not more than $5 \%$ of all fracture cases of long bones. But this entity poses a great problem to both treating surgeon in terms of planning and multiple operative procedures as well as to the patient in terms of pain, functional and psychosocial disability, increased dependency and financial burden.[3] There are ample of causes like anatomical, biochemical, nutritional and hormonal factor that contributes to the occurrence of delayed union/ non-union reported in books and literature. Keeping the insight of all these contributing factors led to the development of multiple techniques reported in literature which breaks the continuum of delayed union and non-union and aid in the early complete union of delayed union long bone fractures.[4,5,6] We are conducting this study to compare the two techniques of reaming for delayed union long bone fractures to show the effectiveness of easy, new and innovative modification of reaming technique on healing rates of delayed union long bone fractures.

\section{Materials and Methods}

A randomized controlled study was conducted in the Department of Orthopaedics, ESI-PGIMSR model hospital, Basaidarapur New delhi. Total of 50 patients clinically and radiologically diagnosed to have delayed union of factures of long bones such as femur, tibia and fibula presented to orthopaedic OPD during the study period of July 2012 to July 2013 were included in the study as cases and divided into two groups with 25 patients in each group on the basis of randomization protocol decided prior to study and taking into consideration inclusion and exclusion criteria mentioned below. 


\section{International Journal of Science and Research (IJSR) \\ ISSN (Online): 2319-7064}

Index Copernicus Value (2013): 6.14 | Impact Factor (2014): 5.611

Table 1: Inclusion and Exclusion Criteria

\begin{tabular}{|c|c|c|}
\hline S. No. & Inclusion criteria & Exclusion criteria \\
\hline 1. & Age 18-60 years & Open fractures \\
\hline 2. & $\begin{array}{c}\text { Delayed union long bone fractures } \\
\text { of lower limb }\end{array}$ & $\begin{array}{c}\text { Pathological } \\
\text { fractures }\end{array}$ \\
\hline 3. & $\begin{array}{c}\text { Delayed union duration up to 5 } \\
\text { months }\end{array}$ & Signs of infection \\
\hline 4. & $\begin{array}{c}\text { Delayed union lower limb fractures } \\
\text { managed previously with nailing }\end{array}$ & $\begin{array}{c}\text { Uncontrolled } \\
\text { diabetes mellitus }\end{array}$ \\
\hline 5. & $\begin{array}{c}\text { Delayed union lower limb fractures } \\
\text { managed conservatively in cast }\end{array}$ & Excessive smoking \\
\hline
\end{tabular}

In group 1 , technique 1 of reaming utilized which includes reaming of proximal fragment first with increasing size of reamer then reaming of distal fragment with increasing size of Reamer. In group 2, technique 2 of reaming utilized which is a classical technique of reaming both the fragments in sequential manner with increasing size of reamers. Both the groups followed by nailing of adequate sizes and predetermined post-operative protocol. Post-operative protocol - Patients in both the groups started with static and dynamic quadriceps exercises from next day of the surgery. Once patients become comfortable, they were allowed to walk with assistive devices (walker) non-weight bearing / toe-touch walking. Suture removal done in all cases at 14 days after thorough inspection of wound. Partial weight bearing with walker started from 3 weeks and progressed to full weight bearing at 6 weeks without walker.

Clinical and radiological evaluation - All the patients in two groups were assessed clinically for pain/tenderness on palpation at fracture site, difficulty in weight bearing, pain/tenderness at the fracture site while weight bearing and radio-logically for fracture healing utilizing radiographs in two orthogonal views. Clinical and radiological assessment done at 3weeks, 6 weeks,3months, 6 months, 9 months, 12 months and 15 months post-operatively.

Both groups were compared for (1) duration from procedure and initiation of bridging callus, and (2) duration from procedure and complete union. Both groups were compared for the (3) number of patients required re-surgery for bone grafting in case of no healing progression or non-union after procedure. Patients who will require re-surgery will be operated with autologous bone grafting procedure from iliac crest.

\section{Results}

Both the groups were demographically similar in terms of age, sex and site of fracture. Out of 50 patients of both groups enrolled in the study, 32 patients with delayed union long bone fracture achieved bone healing/union by the last follow up of 15 months. Total of 18 patients in both groups required re-surgery due to non-progression of fracture healing after the procedure.

Table 2: Demographic Distribution

\begin{tabular}{|c|c|c|c|}
\hline S. No. & Entity & Group 1 & Group 2 \\
\hline 1 & No. of cases & 25 & 25 \\
\hline 2 & Age (mean age in years) & 46 years & 48 years \\
\hline 3 & Sex (M:F) & $18: 7$ & $20: 5$ \\
\hline 4 & Site of fracture (femur : tibia) & $6: 19$ & $4: 21$ \\
\hline 5 & Re-surgery (no. of patients) & 5 & 13 \\
\hline
\end{tabular}

Group 1 showed mean duration of 2 months from procedure and initiation of bridging callus and mean duration of 10 months from procedure and complete union. Only 5 patients in group 1 required re-surgery for bone grafting. While in group 2, mean duration from procedure and initiation of bridging callus found to be 3 months and mean duration from procedure and complete union found to be 12 months. 13 patients in group 2 required re-surgery for bone grafting. There is clinically significant difference found between two groups in terms of re-surgery for bone grafting. Statistical analysis was done using chi-square test and odds ratio.

\section{Discussion}

Fracture healing is a biological process involving cellular response for bone formation which requires mechanical stability, adequate blood supply and good bony apposition. The most productive source of cells that influence osteogenesis is considered to be autologous marrow. The healing response may be negatively affected by the severity of the injury ie. open fractures [7,8], suboptimal surgical fixation and a combination of other indirect factors such as infection [7], smoking [8-10], excessive use of NSAIDS [11], diabetes mellitus $[7,8,12]$, Delay in onset of treatment and initial modality of treatment [13] which can predispose to delayed union/ non-union of long bone fractures. We have included most of these factors in exclusion criteria to rule out the maximum confounding factors we can so that results which we get should be more genuine.

The work of Paley et al [14], Connolly et al [15], Garg et al [16], R bhargava et al [5] showed that bone marrow aspiration from iliac crest and injecting it into the vicinity of fracture site in cases of delayed union long bone fractures helps in achieving early and complete bone healing in most of the cases.

This idea prompt us to formulate this technique of reaming which helps in concentration of marrow around fracture site without doing bone marrow aspiration from iliac crest and additional procedure of injecting bone marrow locally in vicinity of fracture site. On the basis of previous studies on bone marrow injection in fracture vicinity for delayed union long bone fractures, we formulated a technique of reaming which concentrates the bone marrow of long bone proximal fragment around the fracture site which may helps in early healing of fracture.

We have included delayed union long bone fractures of lower limb of up to 5 months duration and excluded all cases with associated risk factors for non-union such as open fractures, infection, cigarette smoking and uncontrolled diabetes so that most of the confounding factors can be excluded and results came out to be more genuine.

We found better results with our new, innovative technique of reaming for delayed union long bones fracture of less than 5 months duration compared to classical technique of reaming. Only 5 patients required re-surgery after our technique of reaming compared to 13 patients in other group who required re-surgery. 


\section{International Journal of Science and Research (IJSR) \\ ISSN (Online): 2319-7064}

Index Copernicus Value (2013): 6.14 | Impact Factor (2014): 5.611

All the 18 cases who required re-surgery with autologous bone grafting from iliac crest in our study healed well at the last follow-up.

The only problem with our new technique of reaming we found was it takes more time during surgery compared to other group as this technique requires two times the number of reaming required for the classical technique of reaming and more amount of radiological exposure compared to other group intra-operatively.

In review of literature we didn't find a single study mentioning this technique of reaming for delayed union long bone fracture of lower limb which is innovated at our institute and this is the only study conducted comparing two techniques of reaming for delayed union long bone fractures.

In conclusion, our study showed better results with technique one of doing the reaming of proximal fragment first with increasing size of reamer then reaming of distal fragment with increasing size of reamer than technique two of reaming both the fragments in sequential manner in terms of speed of bone healing and recovery. But in future this requires a large sample group study with stringent inclusion and exclusion criteria to validate the effectiveness of this new innovative technique.

\section{Compliance with Ethical Standards:}

Funding: not applicable.

\section{Conflict of Interest}

Author 1 to 4 declares that they have no conflict of interest.

\section{Ethical Approval}

All procedures performed in our case report involving human participant were in accordance with the ethical standards of the institutional and/or national research committee and with the 1964 Helsinki declaration and its later amendments or comparable ethical standards.

\section{Informed Consent}

Informed consent was obtained from the patient included in our study. Patient has given consent for the Case report to be published.

\section{References}

[1] Einhorn, T.A. Enhancement of fracture healing. Instr Course Lect.1996; 45:401-416.

[2] Praemer, A.; Furner, S.; Rice, D.P. Musculoskeletal Conditions in the United States. Park Ridge, IL, American Academy of Orthopaedic Surgeons, 1992, pp. 83-124.

[3] Lerner, R.K.; Esterhai, J.L., Jr.; Polomono, R.C.; et al. Psychosocial,Functional and quality of life assessment of patients with posttraumatic Fracture nonunion, chronic refractory osteomyelitis, and lower extremity amputation. Arch Phys Med Rehabil.1991; 72:122-126.
[4] Griffin $\mathrm{XL}^{1}$, Costa $\mathrm{ML}$, Parsons N, Smith $\mathrm{N}$. Electromagnetic field stimulation for treating delayed union or non-union of long bone fractures in adults. Cochrane Database Syst Rev. 2011 Apr 13; (4): CD008471.

[5] Rakesh Bhargava, SS Sankhla, Anil Gupta, RL Changani, and KC Gagal. Percutaneous autologus bone marrow injection in the treatment of delayed or nonunion. Indian J Orthop. 2007 Jan-Mar; 41(1): 67-71.

[6] Griffin $\mathrm{XL}^{1}$, Wallace D, Parsons N, Costa ML. Platelet rich therapies for long bone healing in adults. Cochrane Database Syst Rev. 2012 Jul 11; 7: CD009496.

[7] Frey, C.; Halikus, N.M.; Vu-Rose, T.; Ebramzadeh, E. A review of ankle arthrodesis: Predisposing factors to nonunion. Foot Ankle Int. 1994; 15:581-584.

[8] MH Perlman \& DB Thordarson. Ankle fusion in a high risk population: an assessment of nonunion risk factors. Foot Ankle Int 1999; 20: 491-496.

[9] MA Schmitz et al. Effect of smoking on tibial shaft fracture healing. Clin Orthop. 1999;365: 184-200.

[10]EJ Harvey et al. Deleterious effect of smoking on healing of open tibia-shaft fractures. Am J Orthop 2002; 31: 518-521.

[11]PV Giannoudis et al. Nonunion of the femoral diaphysis. The influence of reaming and non-steroidal antiinflammatory drugs. J Bone Joint Surg Br 2000; 82: 655-658.

[12] Kayal, R. A., Tsatsas, D., Bauer, M. A., Allen, B., AlSebaei, M. O., Kakar, S., Leone, C. W., Morgan, E. F., Gerstenfeld, L. C., Einhorn, T. A. and Graves, D. T. Diminished Bone Formation During Diabetic Fracture Healing is Related to the Premature Resorption of Cartilage Associated With Increased Osteoclast Activity. Journal of Bone and Mineral Research.2007; 22: $560-568$.

[13] Manoj Kumar S, Indu D, Asha KP, Sujith H, Nikhil Narayanan, Nazrin Hameed. Factors associated with delayed union of long bones - a case control study. Kerala Journal of Orthopaedics 2014; 27: 6-9.

[14] Paley D, Young MC, Wiley AM, Fornasier VL, Jackson RW. Percutaneous bone marrow grafting of fracture and bony defects. An experimental study in rabbits. Clin Orthop Relat Res. 1986; 208: 300-12.

[15] Connolly JF, Guse R, Tiedeman J, Dehne R.Autologous marrow injection as a substitute for operative grafting of tibial non unions. Clin Orthop Relat Res. 1991; 266: 259-70.

[16] Garg NK, Gauer S, Sharma S. Percutaneous bone marrow grafting in 20 cases of ununited fractures. Acta Orthop Scand.1993; 64: 671-2. 